\author{
ARTIFICIAL SATELLITES, Vol. 43, No. 1 - 2008 \\ DOI: 10.2478/v10018-009-0003-x
}

\title{
ESTIMATION OF IONOSPHERIC DELAYS IN DUAL FREQUENCY GNSS POSITIONING
}

\author{
Hiroshi Isshiki \\ School of Naval Architecture and Ocean Engineering \\ University of Ulsan \\ Ulsan 680-749, Republic of Korea \\ Tel: $82-52-259-2166$ \\ Fax: 82-52-259-2836 \\ Email: isshiki@dab.hi-ho.ne.jp \\ Jinling Wang \\ School of Surveying \& Spatial Information Systems \\ The University of New South Wales \\ Sydney, NSW 2052, Australia \\ Tel: $\quad 61-2-93854203$ \\ Fax: 61293137493 \\ Email: Jinling.Wang@unsw.edu.au
}

\begin{abstract}
The correct estimation of ionospheric delays is very important in precise satellite-based kinematic positioning, especially over long baselines. In the case of triple frequency system, the ionospheric delays can be estimated from the measurements. However, in the case of dual frequency system, the situation becomes more complicated. The precision of those ionosphere models supplied by the external information source such as JPL IONEX Data is not sufficient, as the high frequency component is neglected. The precision of the low frequency component is not sufficient for the use in long baseline kinematic positioning. On the other hand, the high frequency component can be estimated from the phase range measurements. If the low frequency components are estimated by using an external information source or pseudo-range measurements, more reasonable estimates of ionospheric delays may be possible. In this paper, the estimation using the pseudo-range measurements is discussed. The accuracy of the estimates for ionospheric delays is not sufficient at present because of the bias errors in the pseudo-range measurements. However, if the accuracy of the pseudo-range measurements is improved in future by GPS Modernization and GALILEO, the method would become very promising and convenient, since any external informations are needed and realtime estimation of ionospheric delays becomes possible.
\end{abstract}

Keywords: Multiple Frequency, Ionospheric Delay, Pseudo-range, Pseudo-range Bias.

\section{INTRODUCTION}

The biggest problem in high precision GNSS positioning is the determination of initial phase ambiguity of L1 wave. The factors making the ambiguity determination difficult in the case of long baseline kinematic positioning are due to the satellite errors, the ionospheric delays and the tropospheric delays. The ionospheric delays may be the biggest error source. Better 
positioning performance is expected when GPS Modernization and GALILEO take place in near future (Hatch, 1996, 2006; Han \& Rizos, 1999; Isshiki, 2003a, b).

The present GPS is a dual frequency system. The signals consist of L1 signal of $1.58 \mathrm{GHz}$ and L2 signal of $1.23 \mathrm{GHz}$. It may be impossible to solve the above-mentioned problem completely, since no methods can directly determine in real time both the initial integer phase ambiguity and the ionospheric delay simultaneously. When GPS Modernization is realized and L5 wave of $1.18 \mathrm{GHz}$ is added to the present dual frequency system, the two independent geometry free combination can be obtained for each satellite and receiver combination. Hence, the simultaneous determination of the initial phase ambiguity and the ionospheric delay will become possible theoretically, if the determination of the wide-lane ambiguity by HMW (Hatch-Melbourne-Wübbena) combination is also used. LW (wide-lane) ambiguity may be determined without being assisted by any external sources and without being affected by the ionospheric delay.

In the present paper, a method for the correct estimation of the ionospheric delays in case of the dual frequency system is discussed. The high frequency component of the ionospheric delays can be estimated from the phase range measurements very precisely. If the low frequency components are estimated by using the external information source such as IONEX or pseudo-range measurements, a reasonable estimate of the ionospheric delays may be possible. It has already been discussed in Isshiki (2005) that the estimation of the low frequency components by using the external information source is not sufficient but fairly effective. The estimation using the pseudo-range measurements is discussed in the present paper. Unfortunately, the accuracy is not sufficient at present because of the bias errors in the pseudo-range measurements. However, it is expected that the accuracy of the pseudo-range measurements will be significantly improved in the future and the proposed method would become very promising.

\section{MULTIPLE-FREQUENCY OBSERVATION EQUATIONS AND THE SOLUTION}

Multiple frequency observations are essential for long baseline positioning. Let $t$ refer to time and $P_{\kappa \alpha}^{i}(t)$ and $\Phi_{\kappa \alpha}^{i}(t)$ be the pseudo and phase ranges of $L_{\kappa}$ wave between satellite $i$ and receiver $\alpha . \rho_{\alpha}^{i}(t)$ is the true geometric range. $I_{\alpha}^{i}(t)$ and $T_{\alpha}^{i}(t)$ are the ionospheric and tropospheric delays of $L_{1}$ wave. Then, the double difference observation equations are given as (Isshiki (2003a, b, c; 2004a, b, c)

$$
\begin{gathered}
P_{\kappa \alpha \beta}^{i j}=\rho_{\alpha \beta}^{i j}+\frac{f_{1}^{2}}{f_{\kappa}^{2}} I_{\alpha \beta}^{i j}+T_{\alpha \beta}^{i j}+\bar{e}_{\kappa \alpha \beta}^{i j}+e_{\kappa \alpha \beta}^{i j}, \\
\Phi_{\kappa \alpha \beta}^{i j}=\rho_{\alpha \beta}^{i j}-\frac{f_{1}^{2}}{f_{\kappa}^{2}} I_{\alpha \beta}^{i j}+T_{\alpha \beta}^{i j}+\lambda_{\kappa} N_{\kappa \alpha \beta}^{i j}+\varepsilon_{\kappa \alpha \beta}^{i j},
\end{gathered}
$$

where $\bar{e}_{\kappa \alpha \beta}^{i j}$ is the bias term of the hardware error in pseudo-range which originates from hardware, differs by channel and receiver and can't be cancelled by differencing procedure, $e_{\kappa \alpha \beta}^{i j}$ and $\varepsilon_{\kappa \alpha \beta}^{i j}$ are observation errors of the pseudo and phase ranges, and

$$
\begin{aligned}
& (\bullet)_{\alpha \beta}^{i}=(\bullet)_{\alpha}^{i}-(\bullet)_{\beta}^{i}, \\
& (\bullet)_{\alpha}^{i j}=(\bullet)_{\alpha}^{i}-(\bullet)_{\alpha}^{j},
\end{aligned}
$$




$$
(\bullet)_{\alpha \beta}^{i j}=(\bullet)_{\alpha \beta}^{i}-(\bullet)_{\alpha \beta}^{j}=(\bullet)_{\alpha}^{i j}-(\bullet)_{\beta}^{i j} .
$$

If the errors and hardware bias terms are neglected in the observation equations for $L_{\kappa}$ and $L_{\lambda}$ waves, $\rho_{\alpha \beta}^{i j}(t)+T_{\alpha \beta}^{i j}(t), I_{\alpha \beta}^{i j}, N_{\kappa \alpha \beta}^{i j}$ and $N_{\lambda \alpha \beta}^{i j}$ are expressed in terms of $P_{\kappa \alpha \beta}^{i j}(t)$, $P_{\lambda \alpha \beta}^{i j}(t), \Phi_{\kappa \alpha \beta}^{i j}(t)$ and $\Phi_{\lambda \alpha \beta}^{i j}(t)$.

The initial integer phase ambiguity $N_{W(\kappa, \lambda)_{\alpha \beta}}$ of the wide-lane combination of $L_{\kappa}$ and $L_{\mu}$ waves is obtained as

$$
N_{W(\kappa, \lambda)}^{i j} \equiv N_{\kappa \alpha \beta}^{i j}-N_{\lambda \alpha \beta}^{i j}=\frac{\Phi_{\kappa \alpha \beta}^{i j}(t)}{l_{\kappa}}-\frac{\Phi_{\lambda \beta \beta}^{i j}(t)}{l_{\lambda}}-\frac{f_{\kappa}-f_{\lambda}}{f_{\kappa}+f_{\lambda}}\left(\frac{P_{\kappa \alpha \beta}^{i j}(t)}{l_{\kappa}}+\frac{P_{\lambda \alpha \beta}^{i j}(t)}{l_{\lambda}}\right),
$$

where $f_{\kappa}$ and $l_{\kappa}$ are the frequency and wave length of $L_{\kappa}$ wave. Equation (3) is HMW (Hatch-Melbourne-Wübbena) combination (Hatch, 1982, 1996; Melbourne, 1985; Wübbena, 1985; Isshiki, 2003b). When $\kappa=1$ and $\lambda=2$ in equation (3), $f_{1} \approx 1.58 \mathrm{GHz}$ and $f_{2} \approx 1.23 \mathrm{GHz}$, then $\left(f_{1}-f_{2}\right) /\left(f_{1}+f_{2}\right) \approx 0.124$. So, the error introduced by the pseudo-ranges is reduced to $1 / 10$ of the magnitude. The HMW combination is free from the ionospheric and tropospheric delays. So, the wide-lane ambiguities can be calculated precisely without being affected by the baseline length. Averaging the measurements over epochs can eliminate the random errors effectively.

The wide-lane combination $\Phi_{W(\kappa, \lambda)_{\alpha \beta}}{ }^{i j}(t)$ of $\Phi_{\kappa \alpha \beta}^{i j}(t)$ and $\Phi_{\lambda \alpha \beta}{ }_{\lambda_{\alpha \beta}}^{i j}(t)$ is given as

$$
\begin{aligned}
& \Phi_{W(\kappa, \lambda)}^{i j}(t) \equiv \frac{l_{W(\kappa, \lambda)}}{l_{\kappa}} \Phi_{\kappa \alpha \beta}^{i j}(t)-\frac{l_{W(\kappa, \lambda)}}{l_{\lambda}} \Phi_{\lambda \alpha \beta}^{i j}(t) \\
& =\rho_{\alpha \beta}^{i j}-\left(\frac{l_{W(\kappa, \lambda)}}{l_{\kappa}} \frac{f_{1}^{2}}{f_{\kappa}^{2}}-\frac{l_{W(\kappa, \lambda)}}{l_{\lambda}} \frac{f_{1}^{2}}{f_{\lambda}^{2}}\right) I_{\alpha \beta}^{i j}+l_{W \kappa \lambda}\left(N_{\kappa \alpha \beta}^{i j}-N_{\lambda \alpha \beta}^{i j}\right)+T_{\alpha \beta}^{i j}+\varepsilon_{W(\kappa, \lambda)_{\alpha \beta}}^{i j} \\
& =\rho_{\alpha \beta}^{i j}+\frac{f_{1}^{2}}{f_{\kappa} f_{\lambda}} I_{\alpha \beta}^{i j}+l_{W(\kappa, \lambda)} N_{W(\kappa, \lambda)}{ }^{i j}+T_{\alpha \beta}^{i j}+\varepsilon_{W(\kappa, \lambda)}^{i j},
\end{aligned}
$$

where the frequency $f_{W(\kappa, \lambda)}$ and wave length $l_{W(\kappa, \lambda)}$ of the wide-lane combination are defined as

$$
\frac{f_{W(\kappa, \lambda)}}{c}=\frac{f_{\kappa}-f_{\lambda}}{c}=\frac{1}{l_{\kappa}}-\frac{1}{l_{\lambda}}=\frac{1}{l_{W(\kappa, \lambda)}}
$$

and $\varepsilon_{W(\kappa, \lambda)_{\alpha \beta}}^{i j}$ is the error of $\Phi_{W(\kappa, \lambda)_{\alpha \beta}^{i j}}(t) . c$ is the speed of light in vacuum.

The geometry-free combination $\Phi_{G(\kappa, \lambda)_{\alpha \beta}^{i j}}(t)$ of $\Phi_{\kappa \alpha \beta}^{i j}(t)$ and $\Phi_{\lambda \alpha \beta}^{i j}(t)$ is given as

$$
\begin{aligned}
\Phi_{G(\kappa, \lambda)}^{i j}(t) & \equiv \Phi_{\kappa \alpha \beta}^{i j}(t)-\Phi_{\lambda \alpha \beta}^{i j}(t) \\
& =-\left(\frac{f_{1}^{2}}{f_{\kappa}{ }^{2}}-\frac{f_{1}^{2}}{f_{\lambda}{ }^{2}}\right) I_{\alpha \beta}^{i j}(t)+l_{\kappa} N_{\kappa \alpha \beta}^{i j}-l_{\lambda} N_{\lambda \alpha \beta}^{i j}+\varepsilon_{G(\kappa, \lambda)}^{i j}(t),
\end{aligned}
$$

where $\varepsilon_{G(\kappa, \lambda) \alpha \beta}^{i j}$ is the error of $\Phi_{G(\kappa, \lambda)_{\alpha \beta}^{i j}}(t)$. 


\subsection{Dual frequency case}

For the wide-lane based positioning with a dual frequency system like the present GPS, the ionospheric delay $I_{\alpha \beta}^{i j}$ has to be estimated. The varying component of $I_{\alpha \beta}^{i j}$ may be estimated precisely by the geometry-free combination given by equation (6). The variation of $I_{\alpha \beta}^{i j}$ is obtained as

$$
\left(I_{\alpha \beta}^{i j}(t)-I_{\alpha \beta}^{i j}(0)\right) \approx-\frac{\left(\Phi_{1 \alpha \beta}^{i j}(t)-\Phi_{1_{\alpha \beta}}^{i j}(0)\right)-\left(\Phi_{2 \alpha \beta}^{i j}(t)-\Phi_{2 \alpha \beta}^{i j}(0)\right)}{\left(f_{1} / f_{1}\right)^{2}-\left(f_{1} / f_{2}\right)^{2}} .
$$

Now, $I_{\alpha \beta}^{i j}$ is written as

$$
I_{\alpha \beta}^{i j}(t)=I_{\alpha \beta}^{i j}(0)+\left(I_{\alpha \beta}^{i j}(t)-I_{\alpha \beta}^{i j}(0)\right) .
$$

The first term on the right side of equation (8) must be estimated from the other information included in the observation or supplied from an external source such as JPL IONEX Data (Global estimate of the ionospheric delays).

First, equation (8) is averaged over $N$ epochs, and $I_{\alpha \beta}^{i j}(0)$ is written as

$$
N \cdot I_{\alpha \beta}^{i j}(0)=\sum_{t=0}^{N-1} I_{\alpha \beta}^{i j}(t)-\sum_{t=0}^{N-1}\left(I_{\alpha \beta}^{i j}(t)-I_{\alpha \beta}^{i j}(0)\right)
$$

If the error in the pseudo-range is small, $I_{\alpha \beta}^{i j}(t)$ derived from the geometry-free combination of the pseudo-ranges becomes:

$$
I_{\alpha \beta}^{i j}(t) \approx \frac{P_{1 \alpha \beta}^{i j}(t)-P_{2 \alpha \beta}^{i j}(t)-\bar{e}_{1 \alpha \beta}^{i j}+\bar{e}_{2 \alpha \beta}^{i j}}{\left(f_{1} / f_{1}\right)^{2}-\left(f_{1} / f_{2}\right)^{2}}
$$

and may be used to estimate the first term on the right side of equation (9) as

$$
\sum_{t=0}^{N-1} I_{\alpha \beta}^{i j}(t) \approx \sum_{t=0}^{N-1} \frac{P_{1 \alpha \beta}^{i j}(t)-P_{2 \alpha \beta}^{i j}(t)-\bar{e}_{1 \alpha \beta}^{i j}+\bar{e}_{2 \alpha \beta}^{i j}}{\left(f_{1} / f_{1}\right)^{2}-\left(f_{1} / f_{2}\right)^{2}} .
$$

The hardware bias terms $\bar{e}_{\kappa \alpha \beta}^{i j}$ may neglected if they are sufficiently small, or must be estimated otherwise. Then, equations (7), (8), (9) and (11) give

$$
\begin{array}{r}
I_{\alpha \beta}^{i j}(t)=\frac{1}{N} \sum_{t=0}^{N-1} \frac{P_{1 \alpha \beta}^{i j}(t)-P_{2 \alpha \beta}^{i j}(t)}{\left(f_{1} / f_{1}\right)^{2}-\left(f_{1} / f_{2}\right)^{2}}-\frac{\bar{e}_{1 \alpha \beta}^{i j}-\bar{e}_{2 \alpha \beta}^{i j}}{\left(f_{1} / f_{1}\right)^{2}-\left(f_{1} / f_{2}\right)^{2}} \\
+\frac{1}{N} \sum_{t=0}^{N-1} \frac{\Phi_{1 \alpha \beta}^{i j}(t)-\Phi_{2 \alpha \beta}^{i j}(t)}{\left(f_{1} / f_{1}\right)^{2}-\left(f_{1} / f_{2}\right)^{2}}-\frac{\Phi_{1 \alpha \beta}^{i j}(t)-\Phi_{2 \alpha \beta}^{i j}(t)}{\left(f_{1} / f_{1}\right)^{2}-\left(f_{1} / f_{2}\right)^{2}}, \\
t=0,1,2, \cdots, N-1 .
\end{array}
$$

In the present GPS system, $\kappa=1$ and $\lambda=2, f_{1} \approx 1.58 \mathrm{GHz}$ and $f_{2} \approx 1.23 \mathrm{GHz}$ and $\sigma\left[P_{1 \alpha \beta}^{i j}(t)\right]=\sigma\left[P_{2 \alpha \beta}^{i j}(t)\right] \approx 1 m$. So, the error of $\sum_{t=0}^{N-1} I_{\alpha \beta}^{i j}(t)$ is estimated as $0.22 m$ when $N=100$ $(1 \mathrm{epoch}=30 \mathrm{sec})$. This result is not sufficient for precise positioning. 
Next, instead of the pseudo-range, the ionospheric delay $I_{I O N X \alpha \beta}^{i j}(t)$ supplied by IONEX is used. Then

$$
\sum_{t=0}^{N-1} I_{\alpha \beta}^{i j}(t) \approx \sum_{t=0}^{N-1} I_{I O N X}^{i j} \alpha \beta(t) .
$$

The error of this estimation may be around $0.1 \mathrm{~m}$ (Isshiki, 2004c), which is promising. Then, equations (7), (8), (9) and (13) give

$$
\begin{aligned}
& I_{\alpha \beta}^{i j}(t)=\frac{1}{N} \sum_{t=0}^{N-1} I_{I O N X \alpha \beta}^{i j}(t)+\frac{1}{N} \sum_{t=0}^{N-1} \frac{\Phi_{1 \alpha \beta}^{i j}(t)-\Phi_{2 \alpha \beta}^{i j}(t)}{\left(f_{1} / f_{1}\right)^{2}-\left(f_{1} / f_{2}\right)^{2}} \\
& -\frac{\Phi_{1 \alpha \beta}^{i j}(t)-\Phi_{2 \alpha \beta}^{i j}(t)}{\left(f_{1} / f_{1}\right)^{2}-\left(f_{1} / f_{2}\right)^{2}}, \quad t=0,1,2, \cdots, N-1
\end{aligned}
$$

Similar equations for estimating the ionospheric delays such as equations (12) and (14) may also be obtained as follows (Isshiki, 2006). The geometry free combinations for pseudo and phase ranges between $L_{1}$ and $L_{2}$ waves are given as

$$
\begin{aligned}
P_{1 \alpha \beta}^{i j}(t)- & P_{2 \alpha \beta}^{i j}(t)-\left(\bar{e}_{1 \alpha \beta}^{i j}-\bar{e}_{2 \alpha \beta}^{i j}\right) \\
& =\left(\frac{f_{1}^{2}}{f_{1}^{2}}-\frac{f_{1}^{2}}{f_{2}^{2}}\right) I_{\alpha \beta}^{i j}(t)+e_{G(1,2)}^{i j}(t), \\
\Phi_{1 \alpha \beta}^{i j}(t)- & \Phi_{2 \alpha \beta}^{i j}(t)-l_{2} N_{W(1,2)}^{i j} \alpha \\
= & -\left(\frac{f_{1}^{2}}{f_{1}^{2}}-\frac{f_{1}^{2}}{f_{2}^{2}}\right) I_{\alpha \beta}^{i j}(t)+\left(l_{1}-l_{2}\right) N_{1 \alpha \beta}^{i j}+\varepsilon_{G(1,2)}^{i j}(t),
\end{aligned}
$$

where $N_{W(1,2)_{\alpha \beta}^{i j}}$ is the wide-lane ambiguity defined by equation (3). The unknowns in equation (15) are $I_{\alpha \beta}^{i j}(0), I_{\alpha \beta}^{i j}(1), \cdots, I_{\alpha \beta}^{i j}(N-1)$ and $N_{1 \alpha \beta}^{i j}$. Then, a minimum value problem is presented as:

$$
\begin{aligned}
& F\left(I_{\alpha \beta}^{i j}(0), I_{\alpha \beta}^{i j}(1), \cdots, I_{\alpha \beta}^{i j}(N-1), N_{1 \alpha \beta}^{i j}\right) \\
& \equiv \frac{\sigma_{\varepsilon}^{2}}{\sigma_{e}{ }^{2}} \sum_{t=0}^{N-1}\left\{\left(\frac{f_{1}^{2}}{f_{1}^{2}}-\frac{f_{1}^{2}}{f_{2}^{2}}\right) I_{\alpha \beta}^{i j}(t)-\left[P_{1 \alpha \beta}^{i j}(t)-P_{2 \alpha \beta}^{i j}(t)\right]+\left(\bar{e}_{1 \alpha \beta}^{i j}-\bar{e}_{2 \alpha \beta}^{i j}\right)\right\}^{2} \\
& \quad+\sum_{t=0}^{N-1}\left\{-\left(\frac{f_{1}^{2}}{f_{1}^{2}}-\frac{f_{1}^{2}}{f_{2}{ }^{2}}\right) I_{\alpha \beta}^{i j}(t)+\left(l_{1}-l_{2}\right) N_{1 \alpha \beta}^{i j}-\left[\Phi_{1 \alpha \beta}^{i j}(t)-\Phi_{2 \alpha \beta}^{i j}(t)-l_{2} N_{W(1,2)}^{i j} \alpha\right]\right\}^{2} \\
& =\text { minimun, }
\end{aligned}
$$

where $\sigma_{e}{ }^{2}$ and $\sigma_{\varepsilon}{ }^{2}$ are the variances of $e_{G(1,2)}^{i j}$ and $\varepsilon_{G(1,2)}^{i j}$, respectively The stationary conditions of this minimum value problem give

$$
0=\frac{\partial F}{\partial I_{\alpha \beta}^{i j}(t)}
$$




$$
\begin{aligned}
= & \frac{2 \sigma_{\varepsilon}^{2}}{\sigma_{e}^{2}}\left\{\left(\frac{f_{1}^{2}}{f_{1}^{2}}-\frac{f_{1}^{2}}{f_{2}^{2}}\right) I_{\alpha \beta}^{i j}(t)-\left[P_{1 \alpha \beta}^{i j}(t)-P_{2 \alpha \beta}^{i j}(t)\right]+\left(\bar{e}_{1 \alpha \beta}^{i j}-\bar{e}_{2 \alpha \beta}^{i j}\right)\right\}\left(\frac{f_{1}^{2}}{f_{1}^{2}}-\frac{f_{1}^{2}}{f_{2}^{2}}\right) \\
& \left.-2\left\{-\left(\frac{f_{1}^{2}}{f_{1}^{2}}-\frac{f_{1}^{2}}{f_{2}^{2}}\right) I_{\alpha \beta}^{i j}(t)+\left(l_{1}-l_{2}\right) N_{1 \alpha \beta}^{i j}-\left[\Phi_{1 \alpha \beta}^{i j}(t)-\Phi_{2 \alpha \beta}^{i j}(t)-l_{2} N_{W(1,2)}^{i j}\right)_{\alpha \beta}\right]\right\}\left(\frac{f_{1}^{2}}{f_{1}^{2}}-\frac{f_{1}^{2}}{f_{2}^{2}}\right), \\
0= & \frac{\partial F}{\partial N_{1 \alpha \beta}^{i j}}=2 \sum_{t=0}^{N-1}\left\{-\left(\frac{f_{1}^{2}}{f_{1}^{2}}-\frac{f_{1}^{2}}{f_{2}^{2}}\right) I_{\alpha \beta}^{i j}(t)+\left(l_{1}-l_{2}\right) N_{1 \alpha \beta}^{i j}-\left[\Phi_{1 \alpha \beta}^{i j}(t)-\Phi_{2 \alpha \beta}^{i j}(t)-l_{2} N_{W(1,2)}^{i j} \alpha\right]\right\}\left(l_{1}-l_{2}\right) .
\end{aligned}
$$

From the summation of equation (17) with $t=0,1, \cdots, N-1$, equation (12) is obtained, when $\sigma_{\varepsilon}^{2}$ is much less than $\sigma_{e}{ }^{2}$.

If the following equation is used instead of equation (15a):

$$
I_{I O N X}^{i j} \underset{\alpha \beta}{i j}(t)=I_{\alpha \beta}^{i j}(t)+\varepsilon_{I O N X}^{i j} \underset{\alpha \beta}{i j} .
$$

equation (14) is derived, when $\sigma_{\varepsilon}{ }^{2}$ is much less than $\sigma_{e}{ }^{2}$.

By the way, the hardware errors of the pseudo-ranges cannot be overlooked in estimating the errors involved in the ionospheric delays $I_{\alpha \beta}^{i j}$ estimated by using the pseudo and phase ranges. The errors of the pseudo-ranges are estimated as follows. The three geometry free combinations are obtained from equation (1) as

$$
\begin{aligned}
& P_{1 \alpha \beta}^{i j}(t)-\Phi_{1 \alpha \beta}^{i j}(t)=2 \frac{f_{1}^{2}}{f_{1}^{2}} I_{\alpha \beta}^{i j}(t)-l_{1} N_{1 \alpha \beta}^{i j}+\bar{e}_{1 \alpha \beta}^{i j}+e_{1 \alpha \beta}^{i j}(t)-\varepsilon_{1 \alpha \beta}^{i j}(t), \\
& P_{2 \alpha \beta}^{i j}(t)-\Phi_{2 \alpha \beta}^{i j}(t)=2 \frac{f_{1}^{2}}{f_{2}^{2}} I_{\alpha \beta}^{i j}(t)-l_{2} N_{2 \alpha \beta}^{i j}+\bar{e}_{2 \alpha \beta}^{i j}+e_{2 \alpha \beta}^{i j}(t)-\varepsilon_{2 \alpha \beta}^{i j}(t), \\
& \Phi_{1 \alpha \beta}^{i j}(t)-\Phi_{2 \alpha \beta}^{i j}(t)=-\left(\frac{f_{1}^{2}}{f_{1}^{2}}-\frac{f_{1}^{2}}{f_{2}^{2}}\right) I_{\alpha \beta}^{i j}(t)+l_{1} N_{1 \alpha \beta}^{i j}-l_{2} N_{2 \alpha \beta}^{i j}+\varepsilon_{1 \alpha \beta}^{i j}(t)-\varepsilon_{2 \alpha \beta}^{i j}(t) .
\end{aligned}
$$

If $\varepsilon_{1 \alpha \beta}^{i j}(t)$ and $\varepsilon_{2 \alpha \beta}^{i j}(t)$ are neglected and the ambiguities are known, the unknowns in equation (19) are $I_{\alpha \beta}^{i j}(t), \bar{e}_{1 \alpha \beta}^{i j}+e_{1 \alpha \beta}^{i j}(t)$ and $\bar{e}_{2 \alpha \beta}^{i j}+e_{2 \alpha \beta}^{i j}(t)$. Then, $\bar{e}_{1 \alpha \beta}^{i j}+e_{1 \alpha \beta}^{i j}(t)$. and $\bar{e}_{2 \alpha \beta}^{i j}+e_{2 \alpha \beta}^{i j}(t)$ are given by solving equations (19) as

$$
\begin{gathered}
\bar{e}_{1 \alpha \beta}^{i j}+e_{1 \alpha \beta}^{i j}(t) \approx P_{1 \alpha \beta}^{i j}(t)+\frac{\left(1+\left(f_{1} / f_{2}\right)^{2}\right) \Phi_{1 \alpha \beta}^{i j}(t)-2 \Phi_{2 \alpha \beta}^{i j}(t)}{1-\left(f_{1} / f_{2}\right)^{2}} \\
-\frac{\left(1+\left(f_{1} / f_{2}\right)^{2}\right) l_{1} N_{1 \alpha \beta}^{i j}-2 l_{2} N_{2 \alpha \beta}^{i j}}{1-\left(f_{1} / f_{2}\right)^{2}}, \\
\bar{e}_{2 \alpha \beta}^{i j}+e_{2 \alpha \beta}^{i j}(t) \approx P_{2 \alpha \beta}^{i j}(t)+\frac{2\left(f_{1} / f_{2}\right)^{2} \Phi_{1 \alpha \beta}^{i j}(t)-\left(1+\left(f_{1} / f_{2}\right)^{2}\right) \Phi_{2 \alpha \beta}^{i j}(t)}{1-\left(f_{1} / f_{2}\right)^{2}} \\
-\frac{2\left(f_{1} / f_{2}\right)^{2} l_{1} N_{1 \alpha \beta}^{i j}-\left(1+\left(f_{1} / f_{2}\right)^{2}\right) l_{2} N_{2 \alpha \beta}^{i j}}{1-\left(f_{1} / f_{2}\right)^{2}} .
\end{gathered}
$$




\subsection{Triple frequency case}

In the case of a triple frequency system like GALILEO and the future GPS, two independent wide-lane combinations can be formed (Hatch, 1996, 2006; Han \& Rizos, 1999; Isshiki, 2003a, b). Therefore, we have from equation (4) two independent wide-lane combinations:

$$
\begin{aligned}
\Phi_{W(1,2) \alpha \beta}^{i j}(t) & \equiv \frac{l_{W(1,2)}}{l_{1}} \Phi_{1 \alpha \beta}^{i j}(t)-\frac{l_{W(1,2)}}{l_{2}} \Phi_{2 \alpha \beta}^{i j}(t) \\
& =\rho_{\alpha \beta}^{i j}+\frac{f_{1}^{2}}{f_{1} f_{2}} I_{\alpha \beta}^{i j}+l_{W(1,2)} N_{W(1,2)_{\alpha \beta}}^{i j}+T_{\alpha \beta}^{i j}+\varepsilon_{W(1,2) \alpha \beta}^{i j}, \\
\Phi_{W(2,3)}^{i j} \alpha \beta & \equiv \frac{l_{W(2,3)}}{l_{2}} \Phi_{2 \alpha \beta}^{i j}(t)-\frac{l_{W(2,3)}}{l_{3}} \Phi_{3 \alpha \beta}^{i j}(t) \\
& =\rho_{\alpha \beta}^{i j}+\frac{f_{1}^{2}}{f_{2} f_{3}} I_{\alpha \beta}^{i j}+l_{W(2,3)} N_{W(2,3)_{\alpha \beta}^{i j}}+T_{\alpha \beta}^{i j}+\varepsilon_{W(2,3)_{\alpha \beta}}{ }_{i j},
\end{aligned}
$$

If an ionospheric-free combination is obtained by eliminating the ionospheric delay $I_{\alpha \beta}^{i j}$, an unambiguous observation equation (which does not include ionospheric delay) may be obtained, since the wide-lane ambiguities are determined by the HMW combinations. So, the coordinates may be determined without being affected by the ionospheric delays by solving the equations epoch by epoch (Hatch, 1996, 2006).

On the other hand, we have from equation (6) two independent geometry-free combinations;

$$
\begin{aligned}
& \Phi_{G(1,2){ }_{\alpha \beta}^{i j}}(t) \equiv \Phi_{1 \alpha \beta}^{i j}(t)-\Phi_{2 \alpha \beta}^{i j}(t)=-\left(\frac{f_{1}^{2}}{f_{1}^{2}}-\frac{f_{1}^{2}}{f_{2}^{2}}\right) I_{\alpha \beta}^{i j}(t)+l_{1} N_{1 \alpha \beta}^{i j}-l_{2} N_{2 \alpha \beta}^{i j}+\varepsilon_{G(1,2) \alpha \beta}^{i j}(t), \\
& \Phi_{G(2,3)_{\alpha \beta}^{i j}}(t) \equiv \Phi_{2 \alpha \beta}^{i j}(t)-\Phi_{3 \alpha \beta}^{i j}(t)=-\left(\frac{f_{1}^{2}}{f_{2}^{2}}-\frac{f_{1}^{2}}{f_{3}^{2}}\right) I_{\alpha \beta}^{i j}(t)+l_{2} N_{2 \alpha \beta}^{i j}-l_{3} N_{3 \alpha \beta}^{i j}+\varepsilon_{G(2,3)}^{i j}(t),
\end{aligned}
$$

Furthermore, we have from equation (3)

$$
\begin{aligned}
& N_{W(1,2) \alpha \beta}^{i j}=N_{1 \alpha \beta}^{i j}-N_{2 \alpha \beta}^{i j}, \\
& N_{W(2,3)_{\alpha \beta}^{i j}}=N_{2 \alpha \beta}^{i j}-N_{3 \alpha \beta}^{i j} .
\end{aligned}
$$

From theoretical viewpoint, $I_{\alpha \beta}^{i j}(t), N_{1 \alpha \beta}^{i j}, N_{2 \alpha \beta}^{i j}$ and $N_{3 \alpha \beta}^{i j}$ may then be obtained by solving equations (22) and (23) irrespective of the baseline length, since the wide-lane ambiguities $N_{W(1,2)}^{i j}$ and $N_{W(2,3)_{\alpha \beta}^{i j}}$ are determined by the HMW combinations (Isshiki, 2003a, b).

The above mentioned solution seems very attractive. However, the selection of the frequencies used in the triple frequency system planned now is not suited to the purpose of determining the ionospheric delays and ambiguities, since the inter-frequency differences are not sufficient. If the difference between the frequencies is small, the error is magnified. The multi-path error will cause significant errors in the coordinates. Hatch (2006) has discussed this problem from a quite unique viewpoint. His method may become a breakthrough to the noise problem. However, it must be verified by the real data. Especially, the assumption on the magnitude of the observation errors should be checked. 


\section{NUMERICAL RESULTS IN DUAL FREQUENCY CASE}

\subsection{Japanese data}

In the following numerical calculations, the hardware bias terms $\bar{e}_{1 \alpha \beta}^{i j}$ and $\bar{e}_{1 \alpha \beta}^{i j}$ were neglected, and observation data of fixed stations shown in Table 1 were used. These data were observed by GEONET of GSI (Geographical Survey Institute, Japan) on Dec. 6, 2002 and downloaded from the homepage of GSI. The ionosphere was rather active in 2002. The two pseudo-range signals and the two phase signals of $L_{1}$ and $L_{2}$ waves of GPS are given there. The epoch was 30 seconds, and the data between 00:00:00 UT and 02:00:00 UT were used for the calculations. The coordinates $(x, y, z)$ of the stations and the baseline length $d r$ between the stations shown in Table 1 are very precise ones downloaded from the same site. In the following calculations, the precise orbits of the satellites were used, and the tropospheric delays were estimated by the Saastamoinen model.

Table 1. Station Coordinates $(x, y, z)$ and baseline length $d r$ (downloaded from the homepage of GEONET: Dec. 6, 2002).

\begin{tabular}{|l|l|l|c|c|c|r|r|}
\hline Stn Name & Stn.ID & Abbreb. & $x(m)$ & $y(m)$ & $z \quad(m)$ & $d r \quad(m)$ \\
\hline Sapporo & 950128 & Sppr & -3647449.8988 & 2923169.2544 & 4325315.3884 & 0 \\
\hline Eniwa & 960522 & Enwa & -3667125.8966 & 29088822.6546 & 4318149.1057 & 25349.7028 \\
\hline Ichikawa & 93023 & Ichk & -3967874.2402 & 3340981.7058 & 3699025.1252 & 818216.6565 \\
\hline
\end{tabular}

In Table 2, the wide-lane ambiguities of $L_{1}$ and $L_{2}$ signals calculated by equation (3), that is, the HMW combinations are shown. It is verified that the wide-lane ambiguities could be determined quite successfully regardless of the baseline length by the HMW combinations. Much smaller number of data than the two hour data (240 epochs) are sufficient for obtaining wide-lane ambiguities by HMV combinations as can be checked easily.

Table 2a. Wide-lane ambiguities calculated by the HMW combinations (Sppr-Enwa (BL=abt.25km): Dec. 6, 2002, 00:00:00UT-02:00:00).

\begin{tabular}{|c|c|c|c|c|}
\hline & $((05)-(14))$ & $((06)-(14))$ & $((25)-(14))$ & $((30)-(14))$ \\
\hline $\begin{array}{c}\text { Float } \\
\text { Mean) }\end{array}$ & 2200137.932 & -4995375.995 & -5336259.061 & -562506.032 \\
\hline Fix & 2200138.000 & -4995376.000 & -5336259.000 & -562506.000 \\
\hline Std. Dev. & 0.031 & 0.033 & 0.028 & 0.023 \\
\hline
\end{tabular}

Table 2b. Wide-lane ambiguities calculated by the HMW combinations (Sppr-Ichk (BL=abt.800km): Dec. 6, 2002, 00:00:00UT-02:00:00).

\begin{tabular}{|c|c|c|c|c|}
\hline & $((05)-(14))$ & $((06)-(14))$ & $((25)-(14))$ & $((30)-(14))$ \\
\hline $\begin{array}{c}\text { Float } \\
\text { Mean })\end{array}$ & 3945315.988 & -8337131.946 & -7584052.919 & -181954.946 \\
\hline Fix & 3945316.000 & -8337132.000 & -7584053.000 & -181955.000 \\
\hline Std. Dev. & 0.032 & 0.031 & 0.030 & 0.033 \\
\hline
\end{tabular}




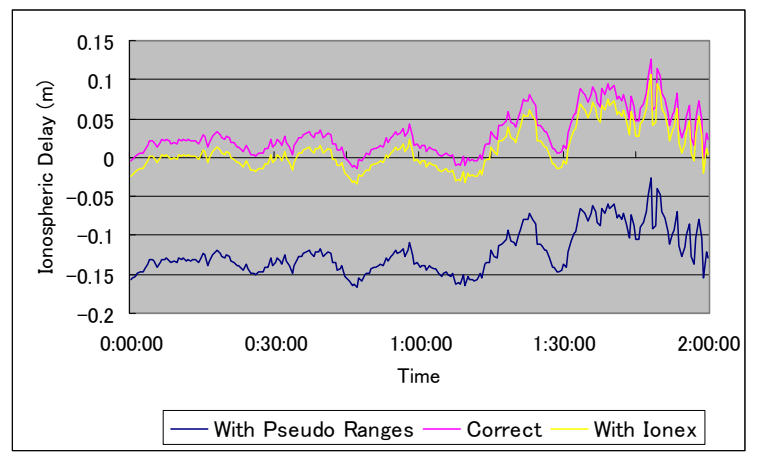

(a) Difference between satellites 5 and 14 .

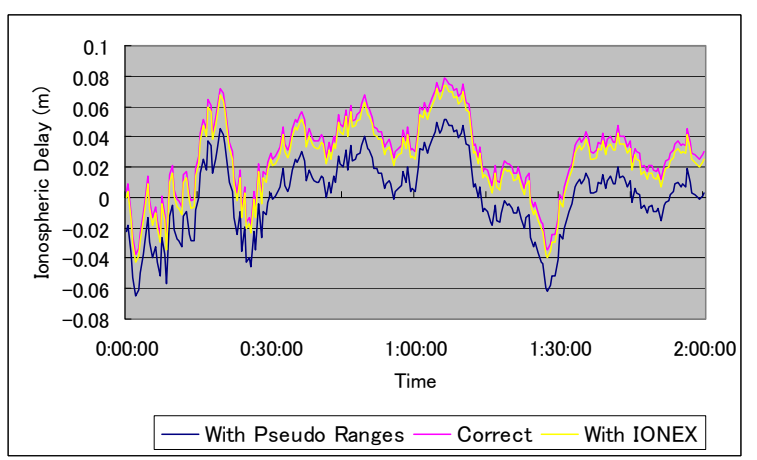

(b) Difference between satellites 6 and 14 .

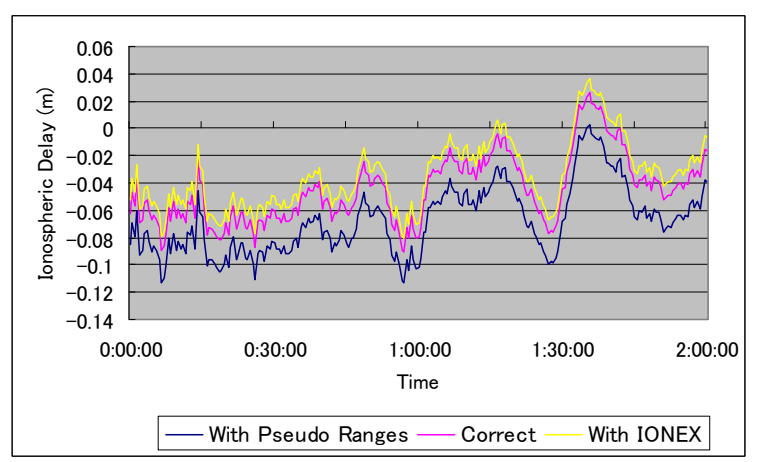

(c) Difference between satellites 25 and 14 .

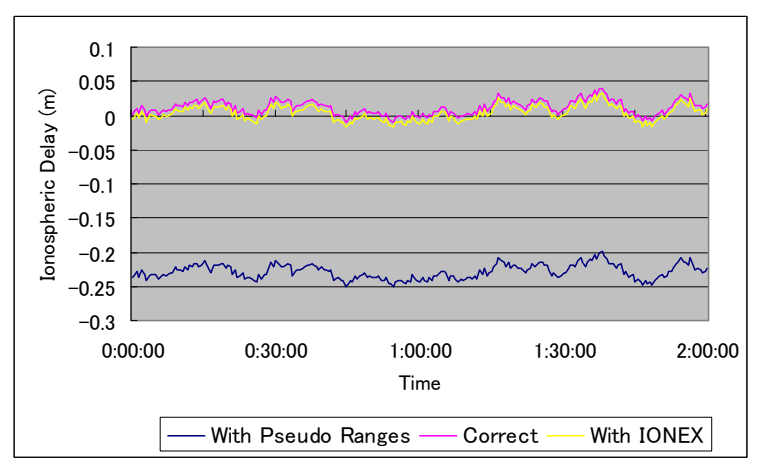

(d) Difference between satellites 30 and 14 .

Fig. 1. Double differenced ionospheric delays between Sppr (Sapporo) and Enwa (Eniwa) (baseline length: about $25 \mathrm{~km}$ ) on Dec. 6, 2002 estimated by phase and pseudo-ranges (blue line) compared with the correct ones (magenta line) and the ones estimated by phase ranges and IONEX (yellow line). 
In Figure 1, the double differenced ionospheric delays between Sapporo and Eniwa (baseline length: about $25 \mathrm{~km}$ ) estimated by phase and pseudo-ranges, that is, equation (12) are compared with the correct ones and the ones estimated by phase ranges and IONEX, that is, equation (14). The correct ones were obtained by using the correct $L_{W(1,2)}$ ambiguities calculated by the HMW combinations, $L_{1}$ ambiguities calculated by the static positioning of the ionosphere free combinations of $L_{1}$ and $L_{2}$ waves and the geometry free combinations of the same waves, since the observation point is fixed in this case.

Equation (20) gives a means to calculate the hardware errors $\bar{e}_{1}$ or $\bar{e}_{1 \alpha \beta}^{i j}$ and $\bar{e}_{2}$ or $\bar{e}_{2 \alpha \beta}^{i j}$ in the pseudo-ranges. The results are shown in Table 3 for Sapporo-Eniwa baseline and in Table 4 for Sapporo-Ichikawa baseline. As shown in Tables 3c and 4c, the differences of averages or biases $\bar{e}_{1}$ and $\bar{e}_{2}$ are very big for some combinations of the satellites. For example, in the case of Table 3c, the differences $\bar{e}_{1}-\bar{e}_{2}$ for ((5)-(14)) and ((30)-(14)) are much bigger than those for ((6)-(14)) and ((25)-(14)). And this corresponds to the big errors in Figures 1(a) and 1(d) as can be understood from equation (12).

Table 3a. The errors $e_{1}$ included in the pseudo-ranges $P_{1}$

(Sppr-Enwa: Dec. 6, 2002, 00:00:00UT-02:00:00).

\begin{tabular}{|r|r|r|r|r|}
\hline & $((5)-(14))$ & $((6)-(14))$ & $((25)-(14))$ & $((30)-(14))$ \\
\hline Average & 0.101786 & 0.00339 & 0.059352 & 0.095625 \\
\hline Std dev & 0.501365 & 0.512642 & 0.410373 & 0.381539 \\
\hline
\end{tabular}

Table 3b. The errors $e_{2}$ included in the pseudo-ranges $P_{2}$

(Sppr-Enwa: Dec. 6, 2002, 00:00:00UT-02:00:00).

\begin{tabular}{|c|r|r|r|r|}
\hline & $((5)-(14))$ & $((6)-(14))$ & $((25)-(14))$ & $((30)-(14))$ \\
\hline Average & 0.003074 & -0.01388 & 0.044528 & -0.05967 \\
\hline Std dev & 0.695572 & 0.798566 & 0.635023 & 0.490122 \\
\hline
\end{tabular}

Table 3c. The averages or biases $\bar{e}_{1}$ and $\bar{e}_{2}$

(Sppr-Enwa: Dec. 6, 2002, 00:00:00UT-02:00:00).

\begin{tabular}{|c|r|r|r|r|}
\hline & $((5)-(14))$ & $((6)-(14))$ & $((25)-(14))$ & \multicolumn{1}{c|}{$((30)-(14))$} \\
\hline $\bar{e}_{1}$ & 0.101786 & 0.00339 & 0.059352 & 0.095625 \\
\hline $\bar{e}_{2}$ & 0.003074 & -0.01388 & 0.044528 & -0.05967 \\
\hline $\bar{e}_{1}-\bar{e}_{2}$ & 0.098711 & 0.017268 & 0.014824 & 0.155298 \\
\hline
\end{tabular}

Similar relationship holds for Sapporo-Ichikawa baseline (about $800 \mathrm{~km}$ ) as shown in Table 4 . So, the bias components in the hardware errors of the pseudo-range are responsible for the poor accuracy of the ionospheric delays as shown by equation (12).

Table 4a. The errors $e_{1}$ included in the pseudo-ranges $P_{1}$

(Sppr-Ichk: Dec. 6, 2002, 00:00:00UT-02:00:00).

\begin{tabular}{|c|c|c|c|c|}
\hline & $((5)-(14))$ & $((6)-(14))$ & $((25)-(14))$ & $((30)-(14))$ \\
\hline Average & -0.01877 & 0.063468 & 0.310909 & -0.00529 \\
\hline Std dev & 0.469117 & 0.43593 & 0.525301 & 0.470828 \\
\hline
\end{tabular}

Table 4b. The errors $e_{2}$ included in the pseudo-ranges $P_{2}$

(Sppr-Ichk: Dec. 6, 2002, 00:00:00UT-02:00:00).

\begin{tabular}{|c|c|c|c|c|}
\hline & $((5)-(14))$ & $((6)-(14))$ & $((25)-(14))$ & $((30)-(14))$ \\
\hline Average & -0.0517 & 0.024594 & -0.36598 & -0.09567 \\
\hline Std dev & 0.91217 & 0.837574 & 0.852099 & 1.154072 \\
\hline
\end{tabular}


Table 4c. The averages or biases $\bar{e}_{1}$ and $\bar{e}_{2}$ (Sppr-Ichk: Dec. 6, 2002, 00:00:00UT-02:00:00).

\begin{tabular}{|c|c|c|c|c|}
\hline & $((5)-(14))$ & $((6)-(14))$ & $((25)-(14))$ & $((30)-(14))$ \\
\hline $\bar{e}_{1}$ & -0.01466 & 0.072276 & 0.318551 & 0.00427 \\
\hline $\bar{e}_{2}$ & -0.0517 & 0.024594 & -0.36598 & -0.09567 \\
\hline $\bar{e}_{1}-\bar{e}_{2}$ & 0.037038 & 0.047681 & 0.684536 & 0.099938 \\
\hline
\end{tabular}

However, they may be improved in future, when the errors in the pseudo-ranges are decreased in the future GPS receiver technology and in the future GNSS system, namely, modernized GPS and GALILEO etc.

\subsection{Australian data}

The data were downloaded from the homepage of ARGN (Australian Regional GNSS Network, http://www.ga.gov.au/bin/data_server/). Two cases of data obtained on Dec. 6, 2002 (0:20:00-1:50:00 GPS time) and Nov. $\overline{6}, 2006$ (0:00:00-1:30:00 GPS time) both for stations Tow2 (Townsville) and Alic (Alice Springs) were analyzed. The baseline length between Tow 2 and Alic is about $1445 \mathrm{~km}$. The stations are static, and the epoch is 30 seconds. Five satellites, 2, 6, 15, 17 and 18 on Dec. 6, 2002, and 3, 7, 14, 15 and 21 on Nov. 6, 2006 were used.

The results are shown in Table 5 for Dec. 6, 2002 and in Table 6 for Nov. 6, 2006. As shown in Tables $5 \mathrm{c}$ and $6 \mathrm{c}$, the differences of averages or biases $\bar{e}_{1}$ and $\bar{e}_{2}$ are very big for some combinations of the satellites. For example, in the case of Table 5c, the differences $\bar{e}_{1}-\bar{e}_{2}$ for (( 2)-(18)) and (( 6)-(18)) are much bigger than those for ((15)-(18)) and $((17)-(18))$. And this corresponds to the big errors in the estimation of the ionospheric delays.

Table 5a. The errors $e_{1}$ included in the pseudo-ranges $P_{1}$

(Tow2-Alic: Dec. 6, 2002, 00:20:00UT-01:50:00).

\begin{tabular}{|c|r|r|r|r|}
\hline & $((2)-(18))$ & $((6)-(18))$ & $((15)-(18))$ & $((17)-(18))$ \\
\hline Average & 0.250629 & 0.126347 & -0.05294 & -0.11449 \\
\hline Std dev & 0.654237 & 0.408212 & 0.365623 & 0.384343 \\
\hline
\end{tabular}

Table $5 \mathrm{~b}$. The errors $e_{2}$ included in the pseudo-ranges $P_{2}$

(Tow2-Alic: Dec. 6, 2002, 00:20:00UT-01:50:00).

\begin{tabular}{|r|r|r|r|r|}
\hline & $((2)-(18))$ & $((6)-(18))$ & $((15)-(18))$ & \multicolumn{1}{|c|}{$((17)-(18))$} \\
\hline Average & -0.54346 & -0.38452 & -0.24985 & -0.2772 \\
\hline Std dev & 0.719435 & 0.409288 & 0.346721 & 0.389228 \\
\hline
\end{tabular}

Table 5c. The averages or biases $\bar{e}_{1}$ and $\bar{e}_{2}$

(Tow2-Alic: Dec. 6, 2002, 00:20:00UT-01:50:00).

\begin{tabular}{|c|r|r|r|r|}
\hline & $((2)-(18))$ & $((6)-(18))$ & $((15)-(18))$ & \multicolumn{1}{c|}{$((17)-(18))$} \\
\hline $\bar{e}_{1}$ & 0.250629 & 0.126347 & -0.05294 & -0.11449 \\
\hline $\bar{e}_{2}$ & -0.54346 & -0.38452 & -0.24985 & -0.2772 \\
\hline $\bar{e}_{1}-\bar{e}_{2}$ & 0.794084 & 0.510865 & 0.196915 & 0.16271 \\
\hline
\end{tabular}

Similar relationship holds for Nov. 6, 2006 (see Table 6). So, the bias components in the hardware errors of the pseudo-range are responsible for the poor accuracy of the ionospheric delays as in the cases of Japanese data. 
Table 6a. The errors $e_{1}$ included in the pseudo-ranges $P_{1}$

(Tow2-Alic: Nov. 6, 2006, 00:00:00UT-01:30:00).

\begin{tabular}{|c|r|r|r|r|}
\hline & $((3)-(14))$ & $((7)-(14))$ & $((15)-(14))$ & $((21)-(14))$ \\
\hline Average & -0.03197 & 0.015311 & -0.04166 & -0.13404 \\
\hline Std dev & 0.571646 & 0.43186 & 0.482065 & 0.627254 \\
\hline
\end{tabular}

Table $6 \mathrm{~b}$. The errors $e_{2}$ included in the pseudo-ranges $P_{2}$ (Tow2-Alic: Nov. 6, 2006, 00:00:00UT-01:30:00).

\begin{tabular}{|c|r|r|r|r|}
\hline & $((3)-(14))$ & $((7)-(14))$ & $((15)-(14))$ & $((21)-(14))$ \\
\hline Average & 0.077447 & 0.022623 & 0.076175 & 0.264468 \\
\hline Std dev & 0.595025 & 0.469606 & 0.556876 & 0.979479 \\
\hline
\end{tabular}

Table 6c. The averages or biases $\bar{e}_{1}$ and $\bar{e}_{2}$

(Tow2-Alic: Nov. 6, 2006, 00:00:00UT-01:30:00).

\begin{tabular}{|c|r|r|r|r|}
\hline & $((3)-(14))$ & $((7)-(14))$ & $((15)-(14))$ & \multicolumn{1}{c|}{$((21)-(14))$} \\
\hline $\bar{e}_{1}$ & -0.03197 & 0.015311 & -0.04166 & -0.13404 \\
\hline $\bar{e}_{2}$ & 0.077447 & 0.022623 & 0.076175 & 0.264468 \\
\hline $\bar{e}_{1}-\bar{e}_{2}$ & -0.10942 & -0.00731 & -0.11784 & -0.3985 \\
\hline
\end{tabular}

\section{CONCLUSIONS}

In the present paper, the DD (double difference) ionospheric delays have been estimated under the present dual frequency system by using both the pseudo and phase ranges. Unfortunately, the accuracy is rather poor because of the big errors involved in pseudo-ranges. It is clarified that the bias components in the hardware errors of the pseudo-range are responsible for the poor accuracy of the ionospheric delays. The same results are obtained both for Japanese and Australian data.

It is expected that the accuracy of the pseudo-range measurements will be significantly improved with future GPS receiver technology and future GNSS systems such as GPS Modernization and GALILEO, and the proposed method will certainly become very promising.

When the present L2 signals are reinforced and opened to commercial use as L2C, the importance of the dual frequency system using L1 and L2C would be increased much. So, if the ionosphere delays are estimated correctly by using dual-frequency-signals without being supported by the external information source, the dual-frequency-based kinematic method such as discussed in the present paper would give a precise and robust method of kinematic positioning and be very important.

If the more precise ionospheric estimation becomes available in future, the precision of the wide-lane positioning may be increased further, and the wide-lane based realtime kinematic positioning discussed in the present paper may become one of the precise and convenient kinematic positioning methods.

\section{REFERENCES}

Isshiki H. (2003a) An application of wide-lane to long baseline GPS measurements (3), ION GPS/GNSS 2003, The Institute of Navigation.

Isshiki H. (2003b) An approach to ambiguity resolution in multi frequency kinematic positioning, Proceedings of 2003 International Symposium on GPS/GNSS, pp. 545-552. 
Isshiki H. (2003c) Long baseline technology for floating body motion measurements in ocean by GPS -Possibility of dual frequency system-, Conference Proceedings The Society of Naval Architects of Japan, Vol. 2, No.2003A-GS2-2, in Japanese.

Isshiki H. (2004a) Long baseline GPS kinematic positioning by wide-lane combination, Conference Proceedings The Society of Naval Architects of Japan, Vol. 3, No.2004S-G2-10, in Japanese.

Isshiki H. (2004b) A long baseline kinematic GPS solution of ionosphere-free combination constrained by widelane combination, OCEANS'04, Kobe, Japan.

Isshiki H. (2004c) Wide-lane Assisted Long Baseline High Precision Kinematic Positioning by GNSS, GNSS 2004, Sydney, Australia.

Isshiki H. (2005) Long Baseline Wide-Lane Kinematic Positioning in Multiple Frequencies, GNSS 2005, Hong Kong.

Isshiki H. (2006a) Estimation of Ionospheric Delays in Dual Frequency Positioning, IGNSS Symposium 2006, Australia.

Isshiki H. (2006b) Estimation of Ionospheric Delays in Dual Frequency Positioning- Future Possibility of Using Pseudo-range Measurements -, 2006 International Symposium on GPS/GNSS, Korea.

Han S., Rizos C. (1999) The Impact of Two Additional Civilian GPS Frequencies on Ambiguity Resolution Strategies, ION NTM 1999, The Institute of Navigation.

Hatch R. (1982) Synergism of GPS code and carrier measurements, Proceedings of the Third International Geodetic Symposium on Satellite Doppler Positioning, New Mexico State University, pp.1213-1232.

Hatch R. (1996) The Promise of a Third Frequency, GPS World, May, 1996, pp. 55-58.

Hatch R. (2006) A New Three-Frequency, Geometry-Free, Technique for Ambiguity Resolution, ION GPS/GNSS 2006, The Institute of Navigation.

Melbourne W. G. (1985) The case for ranging in GPS based geodetic systems, Proceedings 1st International Symposium on Precise Positioning with the Global Positioning System, edited by Clyde Goad, pp. 403-412, U.S. Department of Commerce, Rockville, Maryland.

Wübbena G. (1985) Software developments for geodetic positioning with GPS using TI 4100 code and carrier measurements, Proceedings 1st International Symp. on Precise Positioning with the Global Positioning System, edited by Clyde Goad, pp. 403-412, U. S. Dept. of Commerce, Rockville, Maryland.

Hugeltobler H., Schaer, S., Fridez, P. (2001) Bernese GPS Software Version 4.2, Astronomical Institute, University of Berne.

Received: 2007-12-18,

Reviewed: 2008-08-01,

Accepted: 2008-08-02. 